\title{
MODELOS Y TEORÍAS DE ENFERMERÍA: SUSTENTO PARA LOS CUIDADOS PALIATIVOS
}

\author{
NURSING MODELS AND THEORIES: SUPPORT FOR PALLIATIVE \\ CARE
}

\section{MODELOS E TEORIAS DE ENFERMAGEM: SUPORTE PARA CUIDADOS PALIATIVOS}

\author{
Natalie Figueredo Borda \\ Universidad Católica del Uruguay \\ nafiguer@ucu.edu.uy \\ ORCID: 0000-0002-1508-7837
}

Mirliana Ramírez-Pereira

Universidad de Chile

ORCID: 0000-0003-1439-4162

\author{
Selene Nurczyk \\ Universidad Católica del Uruguay \\ ORCID: 0000-0002-6214-3782
}

\section{Valeria Diaz-Videla}

Universidad de Chile

ORCID:0000-0002-7130-3661

DOI: https://doi.org/10.22235/ech.v8i2.1846

Recibido: 05/02/2019

Aceptado: 04/08/2019

\section{RESUMEN:}

El cuidado es la base de la ciencia de enfermería y puede conceptualizarse como todas las acciones que ayudan a preservar y mantener la vida. Se origina en la necesidad de supervivencia humana en circunstancias adversas, y si bien es inherente a la especie, se ha diferenciado por género. Las necesidades de cuidado familiar, alimentos, ropa y afecto se han relacionado históricamente con las mujeres; en el caso de los hombres, la atención se relaciona con actos que requieren fuerza física y el logro de alimentos a través del trabajo de caza y pesca. La enfermería moderna tomó desde sus orígenes la práctica del cuidado como la base de su disciplina, siendo abordada desde varios aspectos, en los que se incluye: atención, investigación, docencia y gestión. El cuidado es una parte específica de la cultura, los valores y las creencias de las personas, poniendo a la preocupación por los demás como el pilar de la existencia humana. Cuidar es una forma de vivir y de estar en relación con otros, de habitar en el mundo, que es esencial para cualquier adaptación. Desde la perspectiva de la Enfermería en Cuidados Paliativos, hay varios modelos de enfoques para una intervención holística, que combinan aspectos físicos, sociales, psicológicos y espirituales que brindan atención a la familia y a la sociedad en general. El objetivo de este artículo es identificar la utilización de los modelos y teorías de enfermería en los cuidados paliativos.

Palabras clave: Modelo de Enfermería, Teoría de la Enfermería, Cuidados Paliativos. 


\begin{abstract}
:
Care is the foundation of the Nursing Science and can be conceptualized as all actions that help preserve and maintain life. It originates in the need for human survival in adverse circumstances, and while it is inherent to the species, it has been differentiated by gender. Family care, food, clothing and affection needs have been historically linked to women; in the case of men, care was related to acts requiring physical strength and achieving food through hunting and fishing work. Modern Nursing took since its origins care practice as the basis of their discipline, being approached from various aspects, in which it is included; care, research, teaching and management. Care is a specific part of the culture, values and beliefs of people, placing concern for others as the pillar of human existence. Caring is a way of living and of being in relationship with others, to habit in the world, which is essential for any adaptation.From the perspective of Nursing in Palliative Care, there are various models of approaches to a holistic intervention, which combine physical, social, psychological and spiritual aspects that bring care to the family and society. The objective of this article is to identify the use and application of nursing models and theories in palliative care.
\end{abstract}

Keywords: Nursing model, Nursing theory, Palliative Care.

\title{
RESUMO:
}

O cuidado é a base da ciência da enfermagem e pode ser conceituado como todas as ações que ajudam a preservar e sustentar a vida. Origina-se na necessidade de sobrevivência humana em circunstâncias adversas e, embora seja inerente à espécie, foi diferenciada por gênero. As necessidades de cuidado familiar, alimentação, vestuário e afeto têm sido historicamente relacionadas às mulheres. No caso dos homens, a atenção se relaciona com atos que competem fisicamente na obtenção de alimentos através da caça e pesca.A enfermagem moderna cuidou de si mesma como base de sua disciplina, sendo abordada a partir de diversos aspectos, nos quais está inserida; atenção, pesquisa, ensino e gestão. O cuidado é uma parte específica da cultura, valores e crenças das pessoas, colocando a preocupação pelos outros como o pilar da existência humana. Cuidar é um modo de viver e estar em relação com os outros, de viver no mundo, o que é essencial para qualquer adaptação.Na perspectiva da Enfermagem em Cuidados Paliativos, existem vários modelos de abordagens para uma intervenção holística, que combinam aspectos físicos, sociais, psicológicos e espirituais que dão atenção à família e à sociedade em geral. O objetivo deste artigo é conhecer o uso e aplicação dos modelos e teorias específicas da disciplina de enfermagem em cuidados paliativos.

Palavras chave: Modelo de Enfermagem, Teoria de Enfermagem, Cuidados Paliativos.

\section{INTRODUCCIÓN}

La Organización Mundial de la Salud (OMS) define los cuidados paliativos como: "Un enfoque que mejora la calidad de vida de los pacientes y sus familias que enfrentan los problemas asociados con enfermedades mortales, mediante la prevención y el alivio del sufrimiento por medio de la identificación temprana y la evaluación impecable; el tratamiento del dolor y otros problemas físicos, psicosociales y espirituales" (1). La misma enfatiza el trabajo en equipo como pilar de los programas de servicio paliativo, ayudando, entre otras cosas a que el paciente viva lo más activamente posible hasta su fallecimiento (2).

Por otro lado, el Consejo Internacional de Enfermería, considera en su definición de Enfermería que: "abarca el cuidado autónomo y colaborativo de individuos de todas las edades, familias, grupos y comunidades, enfermos o no y en todos los entornos. La enfermería incluye la promoción de la salud, la prevención de enfermedades y el cuidado de personas enfermas, discapacitadas y moribundas. La defensa, la promoción de un entorno seguro, la investigación, la participación en la 
configuración de la política de salud y en la gestión de los pacientes y los sistemas de salud, y la educación también son funciones clave de enfermería" (3).

La finalidad de la Enfermería es el cuidado, el cual se enmarca en acciones e intervenciones con un sólido fundamento científico y humanista, sustentado en modelos teóricos que orientan la disciplina y la práctica profesional. Estos modelos permiten el desarrollo de la profesión ya que conllevan la interacción de un cuerpo de conocimientos que mejoran la práctica de las enfermeras en todas las áreas (4). La práctica clínica basada en la evidencia sitúa a las enfermeras dentro de una disciplina profesional que aporta el fundamento para que las intervenciones sean originales, innovadoras e individualizadas (5). Del mismo modo, los modelos conceptuales de Enfermería constituyen una serie de concepciones que atribuyen una interpretación general en relación al tema de interés para las enfermeras: el cuidado del otro.

La Enfermería en cuidados paliativos se considera una especialidad dentro de la disciplina, por lo que se requiere estar en posesión de una especialización o un máster en el área. La enfermera también debe tener herramientas de pensamiento crítico y de práctica basada en evidencia, entrenamiento en comunicación, educación de pacientes y familia, cuidado espiritual y psicosocial (6). Además, debe tener formación en los cinco roles básicos de la Enfermería; rol clínico, consultoría clínica, administración, educación e investigación ya que constituye el primer nexo entre el paciente, familia y miembros del equipo interdisciplinario de cuidados paliativos (7). En contrapartida, según Hagan, todas las enfermeras deberían ser competentes para cuidados paliativos, independientemente de su entorno o área de trabajo, haciéndolo posible por medio de programas de capacitación en cuidados paliativos adaptables, de pregrado y posgrado, que incluyan habilidades de comunicación, objetivos de atención, y toma de decisiones. Así como crear un entorno que apoye a las enfermeras desde el punto de vista moral, potenciando su capacidad y alcance laboral (8).

Otro aspecto importante del rol de la enfermera tiene relación con la función de protectora de la dignidad de la persona al final de su vida. Según Johnston las personas desean ser tratados con dignidad y deben también tener el derecho de morir con dignidad. El cuidado con dignidad es una intervención que guía a las enfermeras para identificar y otorgar un cuidado adecuado, desde el punto de vista físico, espiritual, emocional y social (9). La conservación de la dignidad puede ser instrumentalizada por medio de acciones específicas enfocadas en el cuidado, de una manera integral que facilite el trabajo del personal de salud (10).

Este tipo de cuidado es altamente demandante para la enfermera, tanto desde el punto de vista emocional como físico. Inevitablemente la propia historia, autoconocimiento y sensibilidades se entrecruzan con el sufrimiento y experiencia del paciente y su familia. De ahí la importancia de estrategias personales e institucionales que se orienten al autocuidado de la enfermera. Sin embargo, la situación del stress emocional de la enfermera se agudiza cuando se contrapone lo que siente con lo que le fue enseñado en su formación profesional, en la cual el modelo educativo insiste en que debe existir un distanciamiento emocional con el usuario y no involucrar sus sentimientos (9).

A partir de una búsqueda de trabajos académicos publicados entre 2012 y 2018 se pudo enfocar y fundamentar la utilización de los modelos enfermeros para la práctica de los cuidados paliativos. Sin embargo, es importante mencionar que a pesar de la importancia reconocida del uso de fundamentos disciplinares en los cuidados, aún son escasas las publicaciones que dan cuenta de esto.

\section{METODOLOGÍA}

El objetivo de esta investigación fue determinar la utilización de teorías y modelos de enfermería en los cuidados paliativos. Dichas investigaciones y experiencias podrían servir como enlace entre la teoría y la práctica de la enfermería en cuidados paliativos. Se realizó una revisión integrativa, la 
cual se comprende como un método cuyo objetivo es el resumen de investigaciones publicadas, de tal forma de obtener nuevas conclusiones sobre un tema de interés (11).

Se consideraron 5 fases; la primera fue elaboración de la pregunta de investigación, en la cual se definió el tema a investigar según una pregunta directriz, la cual se formuló según la estrategia PICo; correspondiendo la "P" al Problema, "I" Fenómeno de interés, y "Co" al contexto, logrando de esta forma una delimitación del problema (12). En esta revisión integrativa la pregunta planteada fue: ¿Qué investigaciones evidencian la utilización de modelos y/o teorías de Enfermería en Cuidados Paliativos entre los años 2012 y $2018 ?$

En la segunda fase se realizó la búsqueda de literatura. Como criterios de inclusión se consideraron artículos en inglés, español y portugués, en los cuales se evidencia la aplicación en los cuidados paliativos de un modelo o teoría de enfermería, publicados entre el 2012 y 2018, en revistas indexadas en Scopus, Scielo y Web of Science, a texto completo y que incorporaron las palabras clave "Nursing model" AND "Palliative care" y "Nursing theory" AND "Palliative care", en el título, resumen o texto. Las bases de datos seleccionadas fueron incluidas por tener acceso a través de los convenios de las universidades participantes De esta búsqueda emergieron 18 artículos que cumplían con los criterios antes mencionados.

En la tercera fase se recolectaron los datos. La recolección de información se hizo según la metodología señalada en la fase anterior, con memos y notas de campo relevantes para la respuesta a la pregunta de investigación. Por otro lado, también se registraron título, autor, país, año de publicación, metodología de investigación, objetivo y resultados.

La cuarta fase considero el análisis de datos. Se realizó el análisis de los artículos, enfatizando en los criterios de inclusión mencionados. Para finalizar se realizó la última fase con la presentación e interpretación de los resultados, los cuales se presentan en el siguiente apartado.

\section{RESULTADOS Y DISCUSIÓN}

Karlsson y equipo observaron que los pacientes en fase terminal presentaron problemas de resorte de Enfermería, tales como déficit de autocuidado, movilidad física afectada, riesgo de alteración de la integridad cutánea, riesgo de infección, nutrición desequilibrada, fatiga, mucosa oral alterada y dolor. Ellos también determinaron que la orientación de un modelo de Enfermería apoya con mayor precisión la identificación de los principales problemas que presentan las personas y sus familias en el área de los cuidados paliativos. De esta manera, el modelo enfermero evidenciaría los recursos necesarios para un cuidado oportuno, siendo fundamental para la organización, planificación y para la disposición de prioridades por parte de Enfermería (13).

Contreras y colaboradores, investigaron las razones y significados que tiene para la enfermera para trabajar con personas en un programa de cuidados paliativos en Chile. En una muestra de 32 participantes se aplicó un cuestionario sociodemográfico y preguntas abiertas. Luego se analizó, por medio del método Delphi. Los resultados señalan que enfermeras plantean que, para trabajar en el área de cuidados paliativos, es necesario poseer compromiso, conocimientos y vocación. Refieren que requieren mayor formación ya que es un área compleja, especialmente en el área de salud mental, donde cobra relevancia el apoyo emocional que se les puede otorgar a este paciente y su familia (14).

Se ha observado que el sufrimiento al final de la vida de las personas institucionalizadas en residencias genera un fuerte impacto en las enfermeras, ya que le produce sentimientos de impotencia e incapacidad de poder intervenir en estas situaciones. Por lo tanto, el cuidado destinado a aliviar el sufrimiento debe involucrar no sólo el manejo de síntomas, sino que también, la respuesta emocional asociadas a la institucionalización de las personas enfermas (15). 
Los resultados anteriormente descritos coinciden con los hallazgos de Pérez y Cibanal. En una investigación de carácter cualitativo desarrollado en México, las narrativas de las enfermeras dan cuenta de que su self personal debe estar separado de su self profesional, los que les genera gran angustia existencial. En sus discursos las enfermeras también plantean que la importancia de apoyo integral al paciente, que involucre a la familia, personal de salud y a la institución. Por lo tanto, los sentimientos y emociones de la enfermera deben estar vinculada o socializada con el resto de los actores, en otras palabras, se comparte el miedo, angustia y la desesperación (16).

Si bien las enfermeras son los miembros del equipo de salud que tienen mayor contacto con los pacientes frente a una enfermedad que limita la vida, éstas tienen pocas competencias en cuidados paliativos siendo esta razón la causa probable que afecte la calidad del cuidado y la calidad de vida de las personas con dichas enfermedades (17).

Entre los trabajos teóricos que se han explorado, uno de los más utilizados ha sido la Teoría del Déficit de Autocuidado de Dorothea Orem, el cual puede aplicarse a diferentes contextos y en particular a los cuidados paliativos. Orem propone una clasificación de situaciones de cuidado de enfermería que comprende siete grupos, y en el grupo final representa la enfermedad que limita la vida. En este caso, el cuidado se centra en la calidad de vida que se ve afectada gravemente, hasta llegar a la etapa final de la vida. El cuidado central pasa a ser el mantenimiento del confort de la persona en dicha etapa (18).

En esa línea, el modelo conceptual de Orem no sólo está orientado a situaciones de final de la vida, sino que puede orientar el cuidado a personas con enfermedades graves y a largo plazo. Teniendo en cuenta el déficit de autocuidado y las necesidades de las personas enfermas hay diferentes tipos de sistemas de enfermería: sistema totalmente compensatorio, parcialmente compensatorio y de apoyo-educativo (18). Este tipo de sistema proporciona una indicación sobre el grado de participación de la persona en su cuidado personal, para implementar, regular o rechazar la atención terapéutica. La persona o el grupo pueden pasar por los diferentes sistemas o activar varios al mismo tiempo (19).

En el sistema parcialmente compensatorio, se requiere el apoyo parcial de la atención de enfermería, por razones de limitación o discapacidad. En este sistema, la enfermera y el paciente interactúan para satisfacer las necesidades de autocuidado de este último. El paciente puede realizar algunas actividades básicas de cuidado personal, como la higiene y el movimiento limitado (4).

Por otro lado, en el sistema totalmente compensatorio, la persona no puede realizar ninguna actividad de autocuidado, lo que se traduce en una dependencia total de enfermería. Un sistema final es el llamado sistema de educación de apoyo. En este sistema, el individuo necesita orientación e instrucción para llevar a cabo el autocuidado, de modo que la enfermera aconseja, apoya y educa al paciente sobre las medidas necesarias para que la persona pueda realizar su autocuidado (4).

Otra teoría utilizada es la Teoría Humanística de Enfermería, la cual destaca el vínculo que se instaura entre la enfermera y la persona que recibe el cuidado. Se trata de un encuentro enfermerapaciente donde ambos actores se sienten afectados (20). Las perspectivas filosóficas de la Teoría Humanística de Enfermería, según Wu \& Volker resultan aplicables a la práctica de enfermería en cuidados paliativos. La utilización de los conceptos básicos de la Teoría Humanística de Enfermería puede proveer una nomenclatura común para las diferentes etapas del Proceso Enfermero en los cuidados paliativos (21).

El vínculo de confianza y apoyo de las enfermeras con la persona en etapa final de vida es fundamental para promover el confort de la persona, alentarlo en la toma de decisiones y fomentar el mantenimiento de la dignidad y la calidad de vida en esta última fase. La enfermería humanística 
se orienta hacia los valores y propósitos de cuidados paliativos, y en este contexto, tanto la enfermera y las personas que reciben cuidado aportan sus propias perspectivas en el encuentro enfermera-paciente. De esta manera, la Teoría Humanística de Enfermería orienta hacia la importancia de cuidar, de desarrollar la empatía y el encuentro enfermera-persona (22).

Los cuidados paliativos favorecen el holismo, la dignidad y la calidad de vida de las personas que se encuentran en etapa avanzada de la enfermedad y sus familias. Figueiredo sostiene que los principios básicos de la Teoría Humanista de Enfermería son apropiados para todos los profesionales que cuidan de personas al final de la vida, ya que pueden aportar una visión para que las enfermeras empaticen y den respuesta a las personas que se encuentran cercanas a su muerte (22).

El modelo de Davies y Oberle se desarrolló con el propósito de describir el componente clínico del rol de la enfermera en el cuidado paliativo, los atributos de enfermería paliativa para la práctica y para los programas de formación. El rol de Enfermería se orienta hacia un ser solidario con múltiples dimensiones: ser capaz de valorar, ser capaz de lograr conexión, ser capaz de capacitar y hacer, ser capaz de encontrar el significado y la preservación de la propia integridad. Este modelo considera que no debe separarse a la enfermera de su self como persona (23). Newton \& McVicar investigaron si los atributos de enfermería descritos en el modelo original aún se aplicaban, pero no encontraron evidencia que demostrara su aplicación en contextos actuales de cuidados paliativos (24).

Otro de los modelos que han sido utilizados en los cuidados paliativos es el Modelo de Callista Roy. El modelo de Adaptación de Roy sostiene que la persona es un sistema abierto y adaptativo que utiliza una secuencia de entrada, procesamiento y salida; que las personas se adaptan en relación con los estímulos internos y externos que la rodean y que responden a los factores que les generan estrés de forma individual, por sus características de cambiantes (25).

Los procesos de afrontamiento que implica el curso y desarrollo de adaptación que las personas llevan a cabo, incluye la utilización de gran cantidad de energía, la cual según Roy podría emplearse hacia la recuperación de la enfermedad. En su teoría, Roy considera a las personas de una manera holística y sostiene que las mismas están constantemente interactuando con el medio ambiente. El concepto de adaptación implica que la persona es un sistema abierto y que responde a estímulos internos y externos. El rol de la enfermera en el cuidado se traduce en la manipulación de los estímulos provenientes del entorno, con el objetivo de acercarlos al campo de afrontamiento positivo de la persona. Se considera la adaptación como la respuesta eficaz a un estímulo (26).

En este escenario, las enfermeras actúan como reguladores externos, y detectan conductas inconsistentes y sus estímulos, a partir de lo cual llevan a cabo el Proceso Enfermero oportuno para eliminar o aliviar el estímulo y así orientar hacia la adaptación (27). Existe evidencia que la aplicación del modelo de Roy ha sido utilizada con éxito en la mejora de calidad de vida de personas con enfermedades crónicas (28). Las investigaciones en el campo de la oncología de los países occidentales revelaron diversos obstáculos para el manejo efectivo del dolor, tales como: miedo a la adicción, miedo al desarrollo de tolerancia, miedo a los efectos secundarios, y las creencias fatalistas (29), los cuales podrían ser explicados a través del Modelo de Adaptación de Roy.

Retomando el modelo de Orem, los autores Desbiens, Gagnon \& Fillion, consideran que dicho modelo parece proporcionar un marco adecuado para guiar el uso de la Teoría Cognitiva Social de Albert Bandura en el área de Cuidados Paliativos para enfermería. Según los autores, quienes proponen esta teoría compartida, ésta delimita la relación terapéutica entre enfermeras y personas con enfermedad avanzada, además de aportar un escenario para el desarrollo de competencias 
enfermeras en cuidados paliativos y la mejora de la calidad asistencial. En la misma línea, Desbiens sostiene que una teoría compartida podría ser útil como marco en la evaluación de programas de formación en cuidados paliativos para Enfermería. Esta teoría otorga un enfoque para guiar a las enfermeras en el desarrollo de competencias para dar cuidados de calidad a los pacientes con enfermedades en etapas terminales de la enfermedad. Involucra conceptos de competencias de enfermería, auto-competencia, intervenciones de enfermería, autocuidado, síntomas físicos y emocionales y calidad de vida. Todos ellos definen el tipo de relación terapéutica que se producirá entre el cuidado paliativo otorgado por la enfermera y el paciente al final de su vida (28).

Sedillo exploró la auto-competencia del cuidado paliativo en los aspectos clínicos, interacción con el paciente y su familia y prioridades en educación, en 25 trabajadores de un servicio hospitalario. Los datos fueron analizados utilizando la prueba estadística no paramétrica Kruskal-Wallis. La mayoría de los participantes seleccionados mencionaron la capacitación pediátrica en los cuidados paliativos como su principal prioridad para la educación futura.

Por otro lado, los resultados apoyan la hipótesis de que los proveedores de cuidado paliativo tienen diferentes niveles de auto-competencia. El personal clínico no mostró mayor auto-competencia en el uso de analgésicos opioides por vía parenteral; uso de analgésicos adyuvantes; evaluación del dolor en el paciente que no responde o confundido; o la evaluación y manejo del dolor pediátrico, cuando se compara con el resto del personal. Todas las habilidades clínicas mencionadas anteriormente son cruciales para el manejo de los síntomas y la atención al final de la vida. Otro aspecto importante de destacar es que el personal sanitario de apoyo: trabajadores sociales, terapistas físicos y trabajadores comunitarios, tienen una mayor auto-competencia al realizar la evaluación básica del dolor y además de una mejor capacidad para explicar el pronóstico de final de la vida con los miembros de la familia. Puede esperarse este resultado, ya que el personal de soporte informó más años de entrenamiento formal en esta área (29).

Tabla 1. Modelos teóricos y su aporte a los Cuidados Paliativos en Enfermería.

\begin{tabular}{|c|}
\hline Modelo Teórico \\
\hline Cómo aporta a los Cuidados Paliativos \\
\hline Teoría del Déficit de Autocuidado de Dorothea Orem \\
\hline $\begin{array}{l}\text { Las situaciones en las que el autocuidado no es suficiente, cuando hay una } \\
\text { enfermedad que limita la vida, y las personas no pueden realizar acciones } \\
\text { de autocuidado para mantener la salud y bienestar, se desarrolla un déficit } \\
\text { permanente de autocuidado. La enfermera ayuda a estos pacientes a } \\
\text { satisfacer sus demandas de autocuidado. }\end{array}$ \\
\hline Teoría Cognitiva Social de Albert Bandura \\
\hline $\begin{array}{l}\text { El desarrollo de conocimientos y competencias para el cuidado de } \\
\text { personas con enfermedades que limitan la vida implica un esfuerzo } \\
\text { sostenido frente a las dificultades que se presentan. La enfermera es capaz } \\
\text { de identificar autopercepción de las competencias específicas para llevar a } \\
\text { cabo la gestión de cuidados de calidad a los pacientes con enfermedades } \\
\text { potencialmente mortales y su familia. }\end{array}$ \\
\hline Teoría Humanística de Enfermería \\
\hline $\begin{array}{l}\text { La relación enfermera-paciente se caracteriza por interacciones orientadas } \\
\text { a favorecer el bienestar y el crecimiento existencial en el contexto del }\end{array}$ \\
\hline
\end{tabular}


mundo vivido. La enfermera cuida al paciente en etapa terminal de su enfermedad, a través de la escucha, de estar con el paciente, y de otras actividades de enfermería. La enfermera gestiona el cuidado teniendo en cuenta la singularidad y libertad del paciente.

Modelo de Davies y Oberle

La valoración de enfermería del paciente en etapa avanzada de la enfermedad está centrada en aspectos humanos. Se establece una conexión profunda entre los profesionales sanitarios y el paciente. Se busca el empoderamiento del paciente orientándose hacia la independencia en el hacer. La enfermera guía a la persona enferma a preservar su integridad y a encontrar significado en lo que le queda de vida y en su preparación para la muerte.

El modelo de Adaptación de Callista Roy

La puesta en marcha de los procesos de afrontamiento implica el desarrollo de la adaptación de las personas a su situación de salud, y un continuo intercambio con el entorno. Las enfermeras pueden operar sobre los estímulos del entorno gestionando el cuidado de los pacientes en etapa avanzada de la enfermedad, orientando la adecuación de la persona a la nueva situación.

Fuente: Elaboración propia (2019)

\section{CONCLUSIONES}

La disciplina enfermera dispone de modelos y teorías aplicables a las diferentes áreas de cuidados. Sin embargo, se evidencia a través de las publicaciones una subutilización de estos. Es necesaria la adquisición de conocimientos en la aplicación de marcos teóricos que orienten el Proceso Enfermero y que estén sustentados con la evidencia, de tal forma que la disciplina de Enfermería pueda optimizar la experiencia del cuidado, mejorar la salud de las poblaciones y reducir el costo per cápita del cuidado (4).

También es importante destacar que la continuidad asistencial se vincula con la satisfacción de las personas que reciben cuidados y con su calidad de vida. El seguimiento apropiado del cuidado de las personas y la respuesta a los complejos problemas de salud en escenarios cambiantes, constituyen un reto que pone a prueba la capacidad de adaptación del cuidado y de sus profesionales.

Las corrientes que han contribuido al progreso de la práctica enfermera se han orientado principalmente a las técnicas de tratamiento de la enfermedad, siguiendo por la satisfacción de las necesidades de las personas enfermas y finalmente orientadas hacia la salud y su vínculo con aspectos sociales y económicos.

Por medio de los modelos conceptuales de Enfermería se proporciona un marco de referencia para la práctica enfermera, centrados en la red conceptual de enfermería conformada por las categorías: persona, entorno, salud y enfermería. El desarrollo teórico de las enfermeras se ha visto subvalorado y algunos de los aspectos que han contribuido a esta realidad están dados porque muchas enfermeras continúan cimentando su práctica en el modelo biomédico, tal como se realizaba en los inicios de la profesión. Esta postura es un obstáculo para desarrollar el pensamiento crítico y el avance del conocimiento enfermero. 
Algunas enfermeras no reconocen la relación entre la teoría y la práctica asistencial existiendo por consiguiente una disociación entre ambos mundos. Aparentemente esto comienza desde la formación de pregrado, ya que los modelos y teorías son enseñados por docentes distintas a las que supervisan o enseñan aspectos clínicos de la Enfermería. Por otro lado, los estudiantes consideran que las teorías sólo se aprenden de los profesores académicos y de la literatura, mientras que los aspectos de atención directa los adquieren y desarrollan junto a los enfermeros clínicos.

Otro aspecto que ha influido en la baja utilización de modelos y teorías de Enfermería es que, en muchos contextos, el cuidado directo de ciertas personas ha sido delegado al personal auxiliar, mientras que las enfermeras se han focalizado en las actividades administrativas, la ejecución de los procesos de normalización y estandarización de la calidad, y el asegurar la realización de indicaciones de otros profesionales, lo cual ha ido en desmedro del desarrollo disciplinar.

Las enfermeras, como profesionales integrales, que cuidan desde una perspectiva holística deben poseer competencias en aspectos conceptuales y técnicos propios de su profesión y disciplina, además de conocimientos, capacidades, habilidades y actitudes necesarias para afrontar las situaciones que se van presentando y que ayuden a mejorar la calidad del cuidado. Enfermería se encuentra en una búsqueda de autogobierno, demostrando al mundo que es una ciencia, y que el cuidado constituye un aporte a la sociedad. Por otro lado, la democratización del conocimiento por medio de internet, los cambios legislativos que involucran a Enfermería en los distintos países del mundo, cautelando el ejercicio, definiendo roles y reconociendo su papel en la mejora de la calidad de vida de las personas, impulsan el desarrollo científico de la disciplina por medio de los modelos y teorías propios.

\section{REFERENCIAS BIBLIOGRÁFICAS}

1. World Health Organization. Global Atlas on Palliative Care At the End of Life (Internet) 2014 Available from: http://www.thewhpca.org/resources/global-atlas-on-end-of-life-care (Accessed: 2016-07-17)

2. Word Health Organization. Key facts (Internet) 2018 Available from: https://www.who.int/news-room/fact-sheets/detail/palliative-care (Accessed: 2019-06-22)

3. International Council of Nursing. Definition of Nursing. (Internet) 2002 Avalaible from: https://www.icn.ch/nursing-policy/nursing-definitions (Accessed: 2019-06-22)

4. Raile M. Nursing Theorists and Their Work. $8^{\mathrm{a}}$ ed. Madrid: Elsevier Mosby; 2015. 829p. DOI: 10.1080/15524256.20 11.593152

5. Hain,D. \& Haras S. Changing Nephrology Nurses' Beliefs about the Value of Evidence-Based Practice and Their Ability to Implement in Clinical Practice. Nephrology Nursing Journal 2015; 42: 563-566. ISSN: 1526744X

6. Bornem T. Palliative Care Nursing. Current problems in cancer . 2011; 35: 351-356. DOI: 10.1016/j.currproblcancer.2011.10.009

7. De Arco-Canoles OdelC, Suarez-Calle ZK. Rol de los profesionales de enfermería en el sistema de salud colombiano. Univ. Salud.2018;20(2):171-182. DOI: http://dx.doi.org/10.22267/rus.182002.121

8. Hagan TL. Nursing's role in leading palliative care: A call to action. Nurs Educ Today. 2018; 61: 216-19. DOI: 10.1016/j.nedt.2017.11.037.

9. Johnston B. Dignity-conserving care in palliative care setting: An integrative review. Journal of Clinical Nursing.2015; 24:1743-1772. DOI: 10.1111/jocn.12791 
10. Östlund U. How to conserve dignity in palliative care: suggestions from older patients, significant others, and healthcare professionals in Swedish municipal care. BMC Palliative Care. 2019; 18:10. Available from: https://doi.org/10.1186/s12904-019-0393-x (Accessed 2019-06-23).

11. Crossetti MGO. Revisão integrativa de pesquisa na enfermagem o rigor cientifico que lhe é exigido (editorial). Rev Gaúcha Enferm., Porto Alegre (RS) 2012 jun;33(2):10-11.

12. Mamédio C, Andrucioli C, Cuce M. Estrategia PICO para la construcción de la pregunta de Investigación y la búsqueda de evidencia. Rev Latino-am Enfermagem. 2007; 15(3): 508-511.

13. Karlsson M. Community nurses' experiences of ethical problems in end-of-life care in the patient's own home. Scandinavian Journal of Caring Sciences.2013; 27:831-838. DOI: $10.1111 / \mathrm{j} .1471-6712.2012 .01087$

14. Contreras S. Los pacientes del programa alivio del dolor y cuidados paliativos: razones y significados para enfermeras/os. (Patients of the pain relief and palliative care programme: reasons and meaning for nurses) Cienc. Enferm. 2016; 22: 47-63. DOI: 10.4067/S071795532016000100005.

15. Dobrina R. An overview of hospice and palliative care nursing models and theories. International Journal of Palliative Care, 2014; 20: 75-81. DOI: 10.12968/ijpn.2014.20.2.75

16. Perez M.E. Perspectiva de la enfermera ante el paciente oncológico en fase terminal en Tampico, Tamaulipas, México. (Perspective from the nurse to the terminal cancer patient in Tampico, Tamaulipas, Mexico) Cultura de los Cuidados. 2011; 30: 52-59 DOI: 10.7184/cuid.2011.30.08

17. Adams J. Nursing Roles and Strategies in End-of-Life Decision Making in Acute Care: A Systematic Review of the Literature. Research and Practice 2011; Article ID 527834, 15 pages. DOI: $10.1155 / 2011 / 527834$

18. Pina P. Self-care: Orem's theoretical contribution to the Nursing discipline and profession Revista de Enfermagem Referência. 2014. 4:157-163 DOI: 10.12707/RIV14081

19. Johnston et al. An exploration of self-management support in the context of palliative nursing: a modified concept analysis BMC Nursing 2014; 13:13:21. DOI: 10.1186/1472-6955-1321

20. Figueiredo de Sá França R. The importance of communication in pediatric oncology palliative care: focus on Humanistic Nursing Theory Rev. Latino-Am. Enfermagem. 2013; 21 :7806. DOI: $10.1590 / \mathrm{S} 0104-11692013000300018$

21. Wu H. L. \& Volker D. L. Humanistic Nursing Theory: application to hospice and palliative care. Journal of advanced nursing.2012; 68: 471-479. DOI: 10.1111/j.1365-2648.2011.05770.

22. Figueiredo de Sá França R. Importance of Palliative Care in Pediatric Oncology. International Archives of Medicine.2016; 9: 1-10. DOI: 10.1590/S0104-11692013000300018.

23. Oberle K \& Davies B. Support and caring: exploring the concepts. Oncology Nursing Forum.1992; 19: 763-767

24. Newton J. Evaluation of the currency of the Davies and Oberle (1990) model of supportive care in specialist and specialised palliative care settings in England. Journal of Clinical Nursing. 2014. 23: 1662-1676 DOI: 10.1111/jocn.12301.

25. Badr Naga B. Roy Adaptation Model: Application of Theoretical Framework. Middle east journal of family medicine, Middle East Journal of Family Medicine.2014; 12: 48-51

26. Fazel Asgarpoor, A. The effect of a care plan based on the Roy Adaptation Model on level of Fatigue in hemodialysis patients. Evidence Based Care.2011. 1: 77-90. DOI: 10.22038/ebcj.2011.3766 
27. Shahed S. Effect of an Educational-Supportive Program Based on Roy Adaptation Model on Marital Satisfaction in Mastectomy Patients Receiving Chemotherapy Journal of Evidence-based Care. 2016; 6: 71-78 DOI: 10.22038/ebcj.2016.6744

28. Desbiens, J. Gagnon. Development of a shared theory in palliative care to enhance nursing competence. Journal of Advanced Nursing.2012; 68: 2113-2124. DOI: 10.1111/j.13652648.2011.05917

29. Sedillo R A pilot study of palliative care provider self-competence and priorities for education in Kenya. Journal of Hospice \& Palliative Nursing. 2015; 17: 356-363. DOI: 10.1097/NJH.0000000000000176 . 